\title{
XAI Models for Quality of Experience Prediction in Wireless Networks
}

\author{
Alessandro Renda, Pietro Ducange, Gionatan Gallo, Francesco Marcelloni \\ Department of Information Engineering, University of Pisa, Largo Lucio Lazzarino 1, 56122 Pisa, Italy \\ Email: alessandro.renda@ing.unipi.it, pietro.ducange@unipi.it, gionatan.gallo@phd.unipi.it, francesco.marcelloni@unipi.it
}

\begin{abstract}
Explainable Artificial Intelligence (XAI) is expected to play a key role in the design phase of next generation cellular networks. As 5G is being implemented and 6G is just in the conceptualization stage, it is increasingly clear that AI will be essential to manage the ever-growing complexity of the network. However, AI models will not only be required to deliver high levels of performance, but also high levels of explainability. In this paper we show how fuzzy models may be well suited to address this challenge. We compare fuzzy and classical decision tree models with a Random Forest (RF) classifier on a Quality of Experience classification dataset. The comparison suggests that, in our setting, fuzzy decision trees are easier to interpret and perform comparably or even better than classical ones in identifying stall events in a video streaming application. The accuracy drop with respect to RF classifier, which is considered to be a black-box ensemble model, is counterbalanced by a significant gain in terms of explainability.
\end{abstract}

Index Terms-Fuzzy Decision Trees, Explainable Artificial Intelligence, Wireless Networks, B5G, 6G, Quality of Experience

\section{INTRODUCTION}

Wireless networks are a fundamental technology of our times: a prime example are mobile networks, which allow billions of people around the world to communicate with each other. Throughout the years, in addition to progressively improving this service, efforts have been made to respond to other needs of modern society, such as digitalization of industrial sectors and machine-to-machine communication. The steps of this advancement have marked the various generations of cellular networks: so, even if today the deployment of $5 \mathrm{G}$ is just in its infancy, industry and academia have already begun to look beyond $5 \mathrm{G}(\mathrm{B} 5 \mathrm{G})$ and to conceive $6 \mathrm{G}$, the sixth generation of cellular networks [1]. The European Union is at the forefront of this challenge with several Horizon 2020 projects, e.g. HEXA- $X^{1}$, DEDICAT $6 G^{2}$ and $6 G$ BRAINS ${ }^{3}$.

The $6 \mathrm{G}$ network, which is expected to be deployed in 2030, will need to handle the increasing volume of connected devices [2]: compared to current networks, it will be much more complex, will involve network coordination schemes,

This work has been partly funded by the European Commission through the H2020 project Hexa-X (Grant Agreement no. 101015956) and by the Italian Ministry of Education and Research (MIUR) in the framework of the CrossLab project (Departments of Excellence).

${ }^{1}$ https://hexa-x.eu/

${ }^{2}$ https://dedicat6g.eu/

${ }^{3}$ https://6g-brains.eu/ and will serve heterogeneous end-user applications, such as holographic communication, high-precision manufacturing and smart transportation [3]. Evidently, such applications impose stringent requirements in terms of communication parameters such as reliability and latency. It is equally clear that, in this context, any sort of performance optimization can hardly be achieved without the adoption of Artificial Intelligence (AI) and Machine Learning (ML) techniques.

As widely emphasized in recently published works [1]-[3], $\mathrm{AI}$ and ML will play a crucial role in designing and optimizing 6G architectures, protocols and operations. Actually, authors in [2] have highlighted that even in the last decade there have been significant research thrusts for the adoption of AI for wireless communication. The literature review presented by the authors shows how AI approaches have been successfully applied at various layers of the OSI model, with applications ranging from channel estimation and prediction to resource management and scheduling. Notably, the vast majority of such thrusts leverage Deep Learning (DL) models, which have proven to be able to achieve unprecedented levels of performance in various application domains. However, DL models and specifically Deep Neural Networks (DNN) are generally considered opaque or black-box models, due to their large number of layers and huge number of parameters [4]: as such, they suffer from lack of explainability.

Explainable Artificial Intelligence (XAI) is gaining a significant momentum, as both users and government entities are placing increasing attention in the trustworthiness of AI. The recently published "Ethics guidelines for trustworthy AI" [5] states that "[...] AI systems and their decisions should be explained in a manner adapted to the stakeholder concerned. Humans need to be aware that they are interacting with an AI system, and must be informed of the system's capabilities and limitations". Therefore, the lack of explainability of the models proposed as technology enablers for cellular networks can seriously undermine their usability and adoption on a large scale. The cruciality of this aspect has been emphasized in [6]: a review of the core methods of AI in wireless network setting highlights that much emphasis has been put into accuracy-oriented solutions with the adoption of sophisticated AI methods (e.g. DNNs, Deep-Q-Networks, ensemble models) that do not provide inherent explainability.

In this context, the adoption of fuzzy ML models can play a crucial role. For instance, Fuzzy Decision Trees (FDTs) 
and Fuzzy Rule Based Classifiers (FRBCs) provide inherent explainability thanks to the linguistic representation of numerical variables, while still delivering competitive level of performance for both classification and regression tasks [7].

In this paper, we carry out a preliminary investigation on the adoption of an XAI model for the task of Quality of Experience (QoE) classification in wireless networks. In the networks of the present and the future, it is not only mandatory to maintain high levels of Quality of Service (QoS) metrics, intended as a set of quantitative measures that characterize the service offered by the network, but also to ensure the fulfillment of QoE metrics, intended as a measure of the enduser satisfaction [8]. Video streaming is an illustrative example where the distinction between QoS and QoE becomes critical: on the one hand, QoS relates to network-centric metrics, such as statistics about network rate and TCP packets delivery [8]; on the other hand, the user-perceived QoE depends on specific factors such as startup delay, rebuffering events and average video quality and its variation [9]. Therefore, an accurate estimation of these quantities, achieved by leveraging information available on the network, may be fundamental for the adoption of countermeasures aimed at avoiding the degradation of end-users' satisfaction.

Pursuing both the accuracy and the explainability of AI models, we present an experimental comparison between classical Decision Trees (DTs) and Fuzzy Decision Trees (FDTs) for the task of QoE classification: specifically, by adhering to the approach recently proposed in [8], we tackle the prediction of stall events in a streaming video application as a multiclass classification problem. DTs and FDTs are further compared with a Random Forest (RF) [10] classifier: as an ensemble of DTs, it typically outperforms individual DTs but lacks explainability. Our main contribution lies in the adoption of highly explainable AI models, namely FDTs in the context of wireless networks. This may represent a preliminary step towards XAI-enabled next generation cellular networks.

The rest of the paper is organized as follows: Section II describes some related works. Section III provides the background on Fuzzy partitions, FDTs and how they feature explainability. Section IV defines the experimental setup. Results are reported and discussed in Section V. Section VI draws some conclusions.

\section{RELATED WORKS}

Maintaining good visual quality for video applications on mobile devices is of utmost importance to both service providers and network designers. However, only in the last few years efforts have been made to improve the performance of classical models by leveraging ML approaches.

Hameed et al. [11] use a classical DT for predicting the perceptual video quality from a set of observable features from the compressed bitstream and the network. The output of the model feeds a QoE control scheme aimed at minimizing network impairment and enhancing the perceptual quality of videos. The experimental analysis is based on a subjective test involving 100 participants and a total number of about
300 videos. Authors in [12] adopt Random Forest to detect the level of stalling from encrypted and unencrypted traffic from a mobile network. Later, in [13], authors predict video starvation resorting to different users' feature, such as channel conditions and number of active users, evaluating the performance of Generalized Linear Model and Support Vector Machine.

Recently, a general framework for QoE prediction has been introduced in [8]: a high-fidelity simulation environment at both network and streaming levels allows the generation of a massive dataset of video sessions, each associated with QoE measurements. The prediction of the stall events is performed with ensemble methods and Bayesian Networks. However, the aspect of model explainability is not addressed by the authors.

\section{BACKGROUND}

In this section we introduce some prelimaries about fuzzy partitions and FDTs. Furthermore, we discuss the explainability of tree-based models.

\section{A. Fuzzy Partitions}

Let $\mathbf{X}=\left\{X_{1}, X_{2}, \ldots, X_{F}\right\}$ be the set of input attributes of a dataset used for classification. Each instance $\mathbf{x}_{\mathbf{k}}=$ $\left\{x_{k, 1}, x_{k, 2}, \ldots, x_{k, F}\right\}$ has an associated label $y_{k}$ which takes values in the set $C=\left\{C_{1}, C_{2}, \ldots, C_{M}\right\}$, with $M$ denoting the number of possible classes.

Consider a generic input attribute $X_{f}$ defined on the universe $U_{f}$ as a bounded interval in $\mathbb{R}$. Let $Z_{f}=$ $\left\{A_{f, 1}, A_{f, 2}, \ldots, A_{f, T_{f}}\right\}$ be a partition over $U_{f}$ consisting of $T_{f}$ fuzzy sets.

In this paper, we adopt strong triangular fuzzy partitions: each fuzzy set $A_{f, i}$ is fully described through a tuple $\left(a_{f, i}\right.$, $\left.b_{f, i}, c_{f, i}\right)$, where $a_{f, i}$ and $c_{f, i}$ correspond to the left and right extremes of the support of $A_{f, i}$, and $b_{f, i}$ to its core. Notably, strong fuzzy partitions have high level of coverage, completeness and complementarity: as a result, they are widely recognized to have a high interpretability [14].

\section{B. Fuzzy Decision Trees}

A DT is a directed acyclic graph where the topmost node is the root of the tree, every internal node represents a test on an input attribute and every terminal node contains instances from one or more class labels. A test performed on an attribute results in a number of branches originating from the node itself. When only two branches are allowed, the tree is said binary, or two-way; otherwise, if more than two branches are allowed, the tree is said multi-way.

Based on the examples of a training set, DT induction is obtained through a hierarchical partition of the feature space. Such a partition is generated recursively and requires the definition of a criterion for the choice of the most discriminating attribute: Gini Index or Information Gain [15] are among the most popular criteria. The first selected attribute is used in the root of the tree. Whenever an attribute is selected, a set of branches and corresponding child nodes are identified. For each child node, a new attribute is selected, considering only the instances of the training set that satisfy the test associated 
with the respective branch. Typically, the procedure terminates when one of the following conditions is met: (i) the node contains only instances of the same class; (ii) the node contains a number of instances lower than a threshold; (iii) the tree has reached a maximum depth; (iv) the value of the quality of a split (e.g. Information Gain) is lower than a threshold. Notably, the learning procedure implicitly assumes that the attributes are categorical: to deal with continuous attributes a discretization step is required either before or along the tree construction. Once the tree is available, it can be used to classify any unlabeled instance $\hat{\mathbf{x}}$. The instance traverses the tree by following a path from the root to a specific leaf: the class $C_{m}$ associated with that leaf will be assigned to $\hat{\mathbf{x}}$. In other words, the path from the root to a leaf generates a rule.

Several works have focused on integrating DTs with concepts from fuzzy set theory, leading to FDTs [16], [17]. Unlike DT, each node of an FDT represents a fuzzy set, rather than a crisp set. Furthermore, each instance can activate different branches thus reaching multiple leaves.

In this paper, we adopt the multi-way FDT (MFDT) described in [17]. In MFDTs, the number of branches originating from an internal node is exactly equal to the number $T_{f}$ of fuzzy sets in the partition of the input attribute $X_{f}$ selected in the node. The learning process of MFDT consits of two steps: first, a strong fuzzy partition is determined on each continuous attribute through a recursive procedure based on the concept of fuzzy entropy; then the FDT is induced from data by using a multiway splitting mechanism based on Fuzzy Information Gain. Since an unlabeled instance $\hat{\mathbf{x}}$ may simultaneously reach multiple leaves by activating multiple paths of the tree, the inference process computes the matching degree of each leaf, i.e. the strength of the activation. In this paper the instance is assigned to the class that corresponds to the maximum association degree, i.e. the product of the matching degree of a specific rule and the weight associated with each class label in the rule. Thus, a single rule can explain how the classification output has been obtained.

\section{How much is a DT explainable?}

The word explainability is often used interchangeably with other terms, such as interpretability and transparency. Some recent works [4], [7] have shed light on the matter, clarifying the differences and similarities among these concepts in the ML context. A new definition of explainability has recently been provided in [4]: "Given a certain audience, explainability refers to the details and reasons a model gives to make its functioning clear or easy to understand." Interpretability consists in the ability to explain how decision have been taken or to provide the meaning in understandable terms to a human [4]. It refers to an inherent property of a model and is strongly related to its transparency, defined as the capability of understanding the structure of the model itself. On the other hand, explainability can be viewed as an active characteristic of a model, denoting any procedure taken for clarifying or detailing its internal functions.
DTs are generally considered among the most inherently explainable classification models [18]. However, as suggested by authors in [18] a distinction should be made between global and local explanations: the former refers to structural properties of the classifiers, such as the tree size, the number of nodes and leaves, and is strictly related to model transparency. The latter is instead associated with the inference process and focuses on how the classification of a single instance is performed. Therefore, it should be noted that the high level of explainability generally accorded to traditional DTs is not actually a granted feature. Specifically, two factors can degrade or jeopardize the explainability of a DT. The first one is the complexity of the model, in terms of the number of nodes and leaves: it should be noted that explainability and accuracy are typically conflicting objectives and thus accuracy-oriented solutions may turn out to be hard to interpret because of their large number of nodes/leaves [7]; structural complexity is therefore primarily related to global explainability. The second factor relates to the semantic interpretability of rules extracted from the tree which should be expressed linguistically, using linguistic terms whose meaning is easily comprehensible [14].

In this context, tools from fuzzy set theory may play a crucial role [4], [7]. First, the linguistic representation of numerical variables allows a direct human interaction, endowing the model with explicit semantic interpretability. Second, the adoption of fuzzy variables allows for better modeling in contexts with some degree of uncertainty.

\section{EXPERIMENTAL SETUP}

In this section we first describe the setup of the QoE classification problem with specific reference to the dataset used in our empirical comparison. Then, we outline the models adopted in our analysis.

\section{A. The QoS-QoE dataset}

We consider the setup presented by authors in [8] as the starting point for our investigation: one of the main contributions of their work lies in the introduction of the QoSQoE dataset ${ }^{4}$. The dataset has been generated by resorting to a fully controllable environment for multimedia streaming simulation: it collects about 69,000 video streaming sessions described as four sets of features, namely context information, QoS metrics, target QoE factors and hidden QoE variables. The overall number of features is 50 .

As agreed upon in the literature [8], [12] one of the most interesting and challenging task enabled by the dataset consists in deriving a mapping between the QoS metrics and the StallLabel variable. The latter is obtained by discretizing the values of Rebuffering Ratio (RR), i.e. a QoE target variable defined as the portion of time spent in stall events. Hence, three classes are obtained as NoStall for $R R=0$, MildStall for $R R \in(0,0.1)$ and SevereStall for $R R \in[0.1,1]$. Since context information and hidden target QoE variables may be unknown to the network controller in real-world scenarios [8],

\footnotetext{
${ }^{4}$ http://jeremie.leguay.free.fr/qoe/
} 
we only consider the 29 QoS metrics as input variables. The class distribution of the 69129 instances of the dataset is as follows: \{NoStall: 51155, MildStall: 17180, SevereStall: 794$\}$. A description of the input attributes is provided in Table I.

TABLE I

INPUT ATTRIBUTES: QOS METRICS AND THEIR DESCRIPTION.

\begin{tabular}{|l|l|}
\hline Name & Description \\
\hline TCP[Output/Input]Packet & Number of TCP packets (In and Out) \\
\hline TCP[Output/Input]Delay & Avg. delay of TCP packets (In and Out) \\
\hline TCP[Output/Input]Jitter & Avg. jitter of TCP packets (In and Out) \\
\hline TCP[Output/Input]Ploss & Loss rate of TCP packets (In and Out) \\
\hline TCPInputRetrans & Packet retransmissions of TCP \\
\hline StdNetworkRate & Standard deviation of the network rate \\
\hline$[x]$ InputRateVariation; $\mathrm{x}$ in & $\begin{array}{l}\mathrm{x}^{\text {th }} \text { quantile for the network rate (mea- } \\
\text { sured in intervals of 2s) }\end{array}$ \\
$\{0,5,10,25,50,75,90,95,100\}$ & $\begin{array}{l}\text { Std. dev. of inter-arrival times of segment } \\
\text { requests }\end{array}$ \\
\hline StdInterATimesReq & $\begin{array}{l}\mathrm{x}^{\text {th }} \text { quantile for the inter-arrival times of } \\
\text { segment requests }\end{array}$ \\
\hline$[\mathrm{x}]$ InterATimesReq; $\mathrm{x}$ in \\
$\{0,5,10,25,50,75,90,95,100\}$
\end{tabular}

\section{B. Classification models and evaluation metrics}

Pursuing high level of explainability of our classification system, we carry out an empirical comparison on the QoSQoE dataset considering two tree-based models: the traditional Binary Decision Tree (BDT) and MFDT. As for BDT, we refer to the scikit-learn Python implementation ${ }^{5}$ of the CART algorithm [15], adopting Information Gain as splitting criterion. As for MFDT, we refer to the Python implementation ${ }^{6}$ of the sequential version of the algorithm presented in [17], which adopts Fuzzy Information Gain as splitting criterion. Furthermore, we evaluate the performance of an RF classifier referring to its scikit-learn Python implementation and adopting the default parameters configuration.

We carry out a twofold analysis: first, we compare the performance of the classifiers on the original dataset; then, we resort to the random undersampling strategy for taming the severe data imbalance that affects the QoS-QoE dataset.

The performance of our models are evaluated in terms of complexity, i.e. number of nodes and number of leaves, and in terms of several evaluation metrics: micro-average F1measure is considered to assess the general performance of the classifier; precision and recall of each class are evaluated to gain a more detailed insight into the ability to identify stalling events. We adopt a 5-fold stratified cross-validation.

Overall, we use two different parameterizations for each of the algorithms by varying the MaxDepth parameter (i.e. maximum depth of the tree). First, we varied the depth of the MFDT and selected two configurations that yield reasonable models, both in terms of complexity and classification metrics. MFDT3 and MFDT-4 refer to MFDTs with maximum depth set to 3 and 4 , respectively. Then, we set the depth of the classical BDT to obtain two binary trees with complexity levels comparable to those of MFDT-3 and MFDT-4, in terms of overall number of nodes. BDT-6 and BDT-11 have maximum depth of 6 and

\footnotetext{
${ }^{5}$ https://scikit-learn.org/

${ }^{6}$ https://bitbucket.org/mbarsacchi/fuzzyml/src/master/
}

11, respectively. Finally, the maximum number of fuzzy sets in the fuzzy partitioning procedure of MFDT has been set to 5: this ensures high level of semantic interpretability thanks to a straightforward labelling of the fuzzy sets. Indeed, in the limit case of five fuzzy sets, we can use the following labels: VeryLow, Low, Medium, High, VeryHigh.

\section{EXPERIMENTAL RESULTS}

Table II summarizes the results obtained with the original dataset. We recall that in all our experiments stratified crossvalidation has been adopted to preserve the actual class frequencies in each train and test fold. We found that the results across folds are relatively stable: thus, we omitted standard deviation values to improve readability. The best value among fuzzy and classical DTs is highlighted in bold. The best overall value is highlighted in bold-italic.

Table IIa suggests that all models deliver acceptable and comparable values of micro-average F1-measure (around $0.85)$. BDTs slightly outperform MFDTs, but they suffer from a modest overtraining that becomes more evident as depth increases. The structural complexity of the models is reported in Table IIb in terms of number of leaves and number of nodes. RF achieves the highest overall performance, but the global complexity of the ensemble model is three orders of magnitude higher than that of MFDTs and BDTs.

To get a thorough insight into the performance of the classifiers, it is important to analyze the precision and the recall achieved on each target class (Table IIc). Furthermore, in our case study, it is crucial to analyse the classifier's ability to recognize stall events, namely MildStall and SevereStall, that represent just the $25 \%$ and the $1 \%$ of the entire dataset, respectively. As expected, all classifiers achieve high precision and recall values on the majority class (NoStall); the values of the metrics are moderately lower for the intermediate class and significantly lower for the minority class. Specifically, MFDTs evidently fail to capture severe stalling events; BDT6 and BDT-11 maintain a fairly high level of precision, but can barely capture $40 \%$ and $57 \%$ of those events, respectively. We argue that the poor performance of MFDTs in recognizing the minority class may be due to the a priori supervised fuzzy partitioning procedure, which likely ignores the contribution of the few instances from the minority class.

Our subsequent analysis was aimed at improving models performance on the least represented classes. A large number of approaches have been proposed to deal with the class imbalance issue [19]. A comprehensive survey of such techniques is beyond the scope of the present work. However, by sticking to data re-balancing techniques, we have empirically observed that the use of oversampling and hybrid approaches do not significantly improve the results of the classifiers, neither used alone nor following undersampling. Ultimately, we opted for random undersampling the majority classes; at each iteration of the cross-validation, the class distribution for the training set is enforced as follows: \{NoStall: 2000, MildStall: 2000, SevereStall: 635$\}$. Indeed, we observed that this value allows 
good performance on the minority class without compromising too much the precision and recall on the other classes.

Results obtained after re-balancing are reported in Table III. As expected, the RF classifier proves to be the most accurate model. MFDTs achieve comparable or better performance than BDTs in terms of micro-average F1-measure; the performance drop w.r.t. RF is just around 2\% for MFDT- 4 and $4 \%$ for MFDT-3. In addition, the overtraining phenomenon is exacerbated in BDTs and especially for BDT-11. Notably, tree complexity did not change significantly after re-balancing (Table IIIb), except for BDT-11 where the number of nodes and leaves approximately halved with respect to the first experiment. Due to re-balancing, all models improve their recall on both MildStall and SevereStall classes (Table IIIc). MFDT4 achieves the absolute highest F1 measure on each class, outperforming all other tree models. MFDT-3 is similarly accurate on the intermediate class, but it is less effective than other models in capturing severe stall events, as witnessed by the lowest recall value. However, it should be noted that it is significantly less complex than MFDT-4 (Table IIIb). As regards BDTs, BDT-6 generally outperforms BDT-11. It achieves the highest recall value on the minority class, but has lower precision than fuzzy trees.

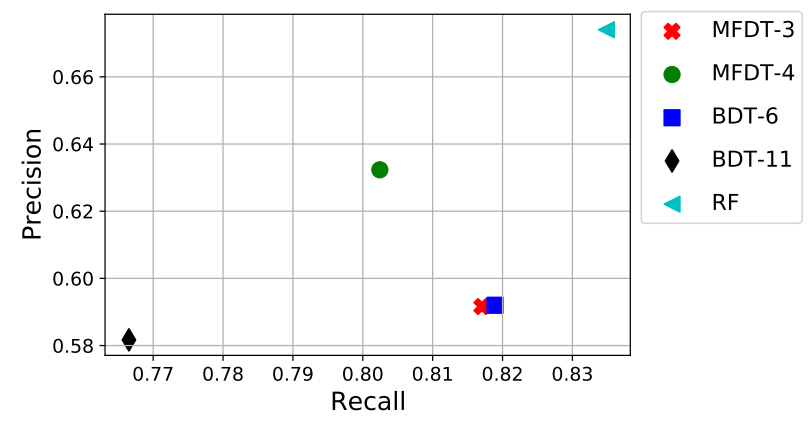

(a) Results on class 1: MildStall

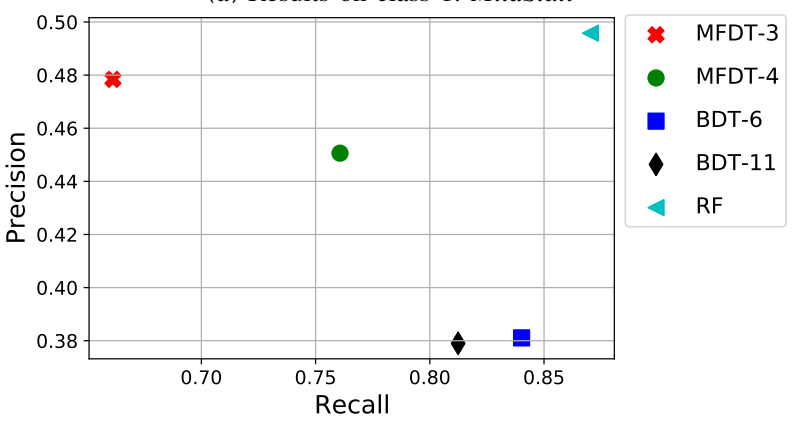

(b) Results on class 2: SevereStall

Fig. 1. Performance of classifiers in terms of precision and recall on the minority classes. Average results.

To sum up, a visual representation of the results is reported in Fig. 1. Each of the five classifiers is represented as a point on the precision-recall plane. We focus only on the least represented classes, namely MildStall (Fig. 1a) and SevereStall (Fig. 1b). Evidently, the best performing algorithms are located in the upper right region in the graphs. RF model outperforms all other models, but it is worth recalling that it suffers from high complexity and poor interpretability. Fig. 1a suggests that the less complex trees, namely BDT-6 and MFDT-3, deliver comparable performance on the MildStall class. As for SevereStall class (Fig. 1b), it can be visually appreciated how the models achieve different trade-offs between precision and recall: if a high recall is desirable, BDT- 6 seems the best choice; conversely, if we want to minimize the number of false alarms, fuzzy models prove to be more appropriate. In particular, MFDT-3 is preferable if high interpretability is a requirement, at the cost of a moderate loss in terms of recall. Interestingly, BDT-6 outperforms BDT-11 with respect to all objectives, namely precision, recall and complexity.

Finally, to assess the semantic interpretability associated with the induced decision trees, we report two examples of rules extracted from MFDT-4 and BDT-6, respectively.

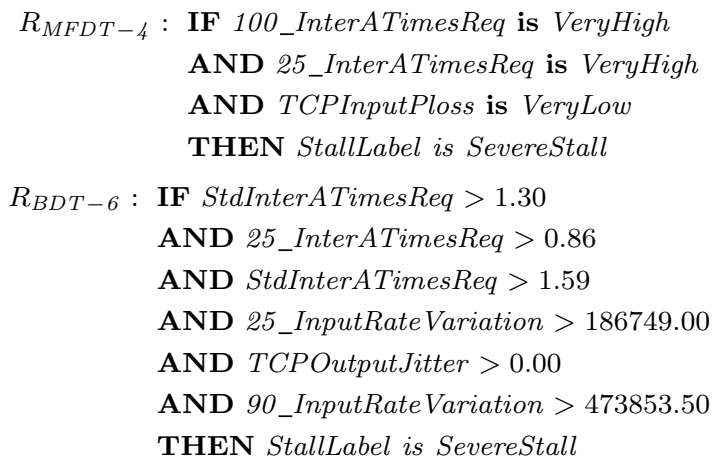

The linguistic representation of numerical variables makes the rules extracted from MFDT-4 easier to interpret than the ones extracted from BDT-6. Notably, in FDTs the partition granularity may vary across attributes, due to the supervised fuzzy partitioning procedure: in the specific case of rule $R_{M F D T-4}$, however, the three attributes in the antecedent have the same number of fuzzy sets (five). The network administrator may benefit from the high level of explainability of the model: for example, it is interesting to notice how an event of severe stalling can be experienced also in presence of a low loss rate of the input TCP packets, for high values of the inter-arrival times of segment requests. As for $R_{B D T-6}$, it is worth noting that attribute StdInterATimesReq is found twice in the rule antecedent. Indeed, the same attribute can be selected more than once in binary trees: this further harms the interpretability of the extracted rules.

\section{CONCLUSION}

In this paper, we have proposed the adoption of fuzzy models for addressing the task of Quality of Experience classification. By leveraging a recently published dataset, conceived for the estimation of the user-perceived video quality, we set up an experimental analysis to compare the performance of multy-way fuzzy Decision Trees and classic binary Decision Trees, with variable maximum depth. A random forest classifier is included in the comparison, as a performance-oriented black-box state-of-the-art approach. As typical of real-world scenarios, the simulated dataset is highly unbalanced and the classes of most concern, related to stall 
TABLE II

RESUlts ON THE ORIGINAL DATASET. Average values.

\begin{tabular}{|c|cc|}
\hline & \multicolumn{2}{|c|}{ F1-measure } \\
\hline & Training & Test \\
\hline MFDT-3 & 0.8443 & 0.8422 \\
MFDT-4 & 0.8493 & 0.8472 \\
BDT-6 & 0.8764 & 0.8678 \\
BDT-11 & $\mathbf{0 . 9 1 5 8}$ & $\mathbf{0 . 8 7 3 7}$ \\
\hline RF & $\mathbf{0 . 9 9 9 9}$ & $\mathbf{0 . 8 9 5 3}$ \\
\hline
\end{tabular}

(a) micro-average F1

\begin{tabular}{|cc|}
\hline \multicolumn{2}{|c|}{ Model Complexity } \\
\hline Leaves & Nodes \\
\hline 88.8 & $\mathbf{1 1 5 . 2}$ \\
331.6 & 438.6 \\
$\mathbf{6 0 . 2}$ & 119.4 \\
740.0 & 1479.0 \\
\hline 337601.0 & 675102.0 \\
\hline
\end{tabular}

(b) Model Complexity

\begin{tabular}{|ccc|ccc|ccc|}
\hline \multicolumn{3}{|c|}{ No Stall } & \multicolumn{3}{c|}{ Mild Stall } & \multicolumn{3}{c|}{ Severe Stall } \\
\hline F1 & Prec. & Recall & F1 & Prec. & Recall & F1 & Prec. & Recall \\
\hline 0.9008 & 0.8881 & 0.9149 & 0.6785 & 0.7017 & 0.6639 & 0.0279 & 0.1846 & 0.0151 \\
0.9032 & 0.9025 & 0.9045 & 0.7015 & 0.6923 & $\mathbf{0 . 7 1 4 9}$ & 0.0257 & 0.1833 & 0.0138 \\
0.9173 & 0.9021 & $\mathbf{0 . 9 3 3 5}$ & 0.7228 & 0.7588 & 0.6938 & 0.5207 & $\mathbf{0 . 7 5 9 9}$ & 0.3994 \\
$\mathbf{0 . 9 2 0 9}$ & $\mathbf{0 . 9 1 0 3}$ & 0.9320 & $\mathbf{0 . 7 3 7 8}$ & $\mathbf{0 . 7 6 5 8}$ & 0.7142 & $\mathbf{0 . 6 0 6 8}$ & 0.6548 & $\mathbf{0 . 5 6 8 1}$ \\
\hline $\mathbf{0 . 9 3 4 8}$ & $\mathbf{0 . 9 1 6 6}$ & $\mathbf{0 . 9 5 3 9}$ & $\mathbf{0 . 7 7 7 7}$ & $\mathbf{0 . 8 2 4 1}$ & $\mathbf{0 . 7 3 7 6}$ & $\mathbf{0 . 6 5 3 6}$ & $\mathbf{0 . 8 6 7 0}$ & 0.5291 \\
\hline
\end{tabular}

(c) Precision and Recall by class on test set.

TABLE III

Results after RE-BALANCing through Random Undersampling. AVERAge Values.

\begin{tabular}{|c|cc|}
\hline & \multicolumn{2}{|c|}{ F1-measure } \\
\hline & Training & Test \\
\hline MFDT-3 & 0.7859 & 0.8119 \\
MFDT-4 & 0.8167 & $\mathbf{0 . 8 3 3 5}$ \\
BDT-6 & 0.8508 & 0.8112 \\
BDT-11 & $\mathbf{0 . 9 4 4 7}$ & 0.8026 \\
\hline RF & $\mathbf{1 . 0}$ & $\mathbf{0 . 8 5 7 5}$ \\
\hline
\end{tabular}

(a) micro-average F1

\begin{tabular}{|cc|}
\hline \multicolumn{2}{|c|}{ Model Complexity } \\
\hline Leaves & Nodes \\
\hline 115.0 & 143.6 \\
396.0 & 500.8 \\
$\mathbf{5 5 . 0}$ & $\mathbf{1 0 9 . 0}$ \\
278.4 & 555.8 \\
\hline 43911.2 & 87722.4 \\
\hline
\end{tabular}

(b) Model Complexity

\begin{tabular}{|ccc|ccc|ccc|}
\hline \multicolumn{3}{|c|}{ No Stall } & \multicolumn{3}{c|}{ Mild Stall } & \multicolumn{3}{c|}{ Severe Stall } \\
\hline F1 & Prec. & Recall & F1 & Prec. & Recall & F1 & Prec. & Recall \\
\hline 0.8712 & 0.9396 & 0.8125 & 0.6856 & 0.5916 & 0.8171 & 0.5546 & $\mathbf{0 . 4 7 8 4}$ & 0.6613 \\
$\mathbf{0 . 8 9 0 7}$ & 0.9415 & $\mathbf{0 . 8 4 5 1}$ & $\mathbf{0 . 7 0 7 1}$ & $\mathbf{0 . 6 3 2 3}$ & 0.8024 & $\mathbf{0 . 5 5 9 0}$ & 0.4506 & 0.7607 \\
0.8731 & $\mathbf{0 . 9 5 0 4}$ & 0.8082 & 0.6862 & 0.5920 & $\mathbf{0 . 8 1 8 9}$ & 0.5235 & 0.3811 & $\mathbf{0 . 8 4 0 2}$ \\
0.8689 & 0.9311 & 0.8146 & 0.6611 & 0.5817 & 0.7665 & 0.5152 & 0.3790 & 0.8124 \\
\hline $\mathbf{0 . 9 0 7 0}$ & $\mathbf{0 . 9 5 3 7}$ & $\mathbf{0 . 8 6 4 9}$ & $\mathbf{0 . 7 4 5 2}$ & $\mathbf{0 . 6 7 4 0}$ & $\mathbf{0 . 8 3 4 9}$ & $\mathbf{0 . 6 2 9 1}$ & $\mathbf{0 . 4 9 5 8}$ & $\mathbf{0 . 8 7 0 3}$ \\
\hline
\end{tabular}

(c) Precision and Recall by class on test set. events, are poorly represented. Nevertheless the adoption of rebalancing techinques significantly improves the performance of the classifiers. The results are analyzed with respect to two main dimensions: on the one hand, we inspect the precision and recall per class achieved by each model, to assess the effectiveness in capturing stalling events; on the other hand, we examine the model complexity, which is directly related to their explainability. Results suggest that, even without any further optimization procedure (e.g. based on evolutionary algorithm), fuzzy decision trees achive competitive values of precision, recall and overall micro-average F1-measure. They also feature higher semantic interpretability than binary crisp trees thanks to the linguistic representation of input variables. DTs are generally less accurate than RF classifier, which, on the other hand, does not feature interpretability. The analysis performed is based on aggregate QoS and QoE metrics: in the near future, we aim to evaluate how inherently explainable approaches, such as fuzzy decision trees and fuzzy rule-based systems, can be used to predict QoE target variables in near real-time over the input data stream. Moreover, multi-objective evolutionary algorithms will be explored for concurrently optimizing the accuracy and the complexity of the fuzzy decision trees.

\section{REFERENCES}

[1] K. B. Letaief, W. Chen, Y. Shi, J. Zhang, and Y.-J. A. Zhang, "The roadmap to 6G: AI empowered wireless networks," IEEE Commun. Mag., vol. 57, no. 8, pp. 84-90, 2019.

[2] R. Shafin, L. Liu, V. Chandrasekhar, H. Chen, J. Reed, and J. C. Zhang, "Artificial intelligence-enabled cellular networks: A critical path to beyond-5G and 6G," IEEE Wireless Commun., vol. 27, no. 2, pp. 212-217, 2020.

[3] K. Sheth, K. Patel, H. Shah, S. Tanwar, R. Gupta, and N. Kumar, "A taxonomy of AI techniques for 6G communication networks," Comput. Commun., vol. 161, pp. 279-303, 2020.

[4] A. B. Arrieta, N. Díaz-Rodríguez, J. Del Ser, A. Bennetot, S. Tabik, A. Barbado, S. García, S. Gil-López, D. Molina, R. Benjamins et al., "Explainable Artificial Intelligence (XAI): Concepts, taxonomies, opportunities and challenges toward responsible AI," Inf. Fusion, vol. 58, pp. $82-115,2020$.
[5] "Ethics Guidelines for Trustworthy AI, Technical Report," 2019, European Commission. High Level Expert Group on AI. https://ec.europa. eu/digital-single-market/en/news/ethics-guidelines-trustworthy-ai.

[6] W. Guo, "Explainable Artificial Intelligence for 6G: Improving Trust between Human and Machine," IEEE Commun. Mag., vol. 58, no. 6, pp. 39-45, 2020.

[7] A. Fernandez, F. Herrera, O. Cordon, M. J. del Jesus, and F. Marcelloni, "Evolutionary fuzzy systems for explainable artificial intelligence: Why, when, what for, and where to?" IEEE Comput. Intell. Mag., vol. 14, no. 1, pp. 69-81, 2019.

[8] V. Vasilev, J. Leguay, S. Paris, L. Maggi, and M. Debbah, "Predicting QoE factors with machine learning," in 2018 IEEE Int'l Conf. on Communications (ICC). IEEE, 2018, pp. 1-6.

[9] X. Yin, A. Jindal, V. Sekar, and B. Sinopoli, "A control-theoretic approach for dynamic adaptive video streaming over HTTP," in Proc. of the 2015 ACM Conf. on Special Interest Group on Data Communication, 2015, pp. 325-338.

[10] L. Breiman, "Random forests," Machine learning, vol. 45, no. 1, pp. 5-32, 2001.

[11] A. Hameed, R. Dai, and B. Balas, "A decision-tree-based perceptual video quality prediction model and its application in FEC for wireless multimedia communications," IEEE Trans. Multimedia, vol. 18, no. 4, pp. 764-774, 2016.

[12] G. Dimopoulos, I. Leontiadis, P. Barlet-Ros, and K. Papagiannaki, "Measuring video QoE from encrypted traffic," in Proc. of the 2016 Internet Measurement Conf., 2016, pp. 513-526.

[13] Y.-T. Lin, E. M. R. Oliveira, S. B. Jemaa, and S. E. Elayoubi, "Machine learning for predicting QoE of video streaming in mobile networks," in 2017 IEEE Int'l Conf. Communications (ICC). IEEE, 2017, pp. 1-6.

[14] M. J. Gacto, R. Alcalá, and F. Herrera, "Interpretability of linguistic fuzzy rule-based systems: An overview of interpretability measures," Inf. Sci., vol. 181, no. 20, pp. 4340-4360, 2011.

[15] L. Breiman, J. Friedman, C. J. Stone, and R. A. Olshen, Classification and regression trees. CRC press, 1984.

[16] Y.-1. Chen, T. Wang, B.-s. Wang, and Z.-j. Li, "A survey of fuzzy decision tree classifier," Fuzzy Inf. Eng., vol. 1, no. 2, pp. 149-159, 2009.

[17] A. Segatori, F. Marcelloni, and W. Pedrycz, "On distributed fuzzy decision trees for big data," IEEE Trans. Fuzzy Syst., vol. 26, no. 1, pp. 174-192, 2017.

[18] J. M. Alonso, P. Ducange, R. Pecori, and R. Vilas, "Building explanations for fuzzy decision trees with the expliclas software," in 2020 IEEE Int'l Conf. on Fuzzy Systems (FUZZ-IEEE). IEEE, 2020, pp. 1-8.

[19] V. López, A. Fernández, S. García, V. Palade, and F. Herrera, "An insight into classification with imbalanced data: Empirical results and current trends on using data intrinsic characteristics," Inf. Sci., vol. 250, pp. 113-141, 2013. 